\title{
Investigating the Efficacy of Biocides Used in Tanta University Hospitals on Antibiotic Susceptibility and Virulence of Bacterial Isolates
}

\author{
Engy Elekhnawy, Tarek Elbanna, Ahmed Abdelaziz and Fatma Sonbol \\ Pharmaceutical Microbiology, Faculty of Pharmacy, Tanta University, Tanta, Egypt
}

\section{III}

Background: Bacterial resistance to antibiotic is a serious challenge. There is a need to study the impact of biocides on the emergence of this resistance. Materials and Methods: A total of 1000 samples including 600 clinical samples compromising urine (58), blood (166), sputum (247), stool (36), and wound pus (93) plus 400 environmental samples were collected from different departments of Tanta University Hospitals, Egypt. All samples were cultured on nutrient agar, then stained by Gram stain and examined microscopically. Gram negative $(n=485)$ and Gram-positive bacteria $(n=115)$ were subjected to biochemical identification and then confirmed by culture on $\mathrm{Hi}$ chrome agar medium. The susceptibility of all the recovered bacterial isolates $(n=600)$ to different antibiotics and different biocides (BAC, TCS, phenol, hydrogen peroxide, chlorohexidine and citric acid) was performed using agar dilution and broth dilution methods, respectively. Results: The increase in antibiotics MICs was relatively more pronounced in Escherichia coli $(n=78)$, Klebsiella pneumoniae $(n=50)$, Proteus mirabilis $(n=31)$ and Pseudomonas aeruginosa $(n=43)$ isolates after adaptation to benzalkonium chloride (BAC) and triclosan (TCS). After adaptation, multiple observations were recorded: 1) BAC and TCS significantly increased $(p<0.05)$ efflux activity in E. coli and K. pneumoniae isolates, respectively; 2) marked increases in the expression of the efflux pump genes acrB and yihV, mdfA; and norE genes; 3) significant decreases in the membrane integrity and permeability; 4) morphological modifications ranged from deformed cells, with individual bumps, grooves, ridges, and cavities, to overall cell surface wrinkling; 5) increases in the average adhesion index; and 6) increases in the $\mathrm{HI}$ and in intl and intll genes were detected in the tested isolates. Conclusion: There is a potential of certain biocides to contribute in the emergence of antibiotic resistance in bacteria. This might have a role in the detection of the relatively high percentage of the multidrug resistant bacterial isolates in the present work.

Keywords: Adaptation; Antibiotics; Biocides; Efflux; Membrane; Resistance

Editor-in-Chief: Prof. M.L. Salem, PhD - Article DOI: 10.21608/jcbr.2021.57805.1117 